\title{
Real-time Internet of Things Architecture for Wireless Livestock Tracking
}

\author{
Biljana Risteska Stojkoska, Dijana Capeska Bogatinoska, Gerhard Scheepers, and Reza Malekian
}

\begin{abstract}
Automatic livestock tracking is necessary for countries facing stock theft problems, like South Africa and Kenya. This paper presents a conceptual design of architecture for real-time wireless livestock tracking based on Internet of Things paradigm. It is a hierarchical model consisting of three building blocks, where the first block is represented with wireless sensor network. Additionally, we have developed a low-power device for livestock tracking in an outdoor environment. The animal tracking device (AnTrack) is self-sustainable with a watertight solar panel(s), designed as a collar to be worn by the animals. A detailed analysis of the AnTrack power consumption proves that the device is capable to generate enough supply power, even when there is no sunshine for a week. This device can be used as a robust building block of future real-time Internet of Things livestock tracking solutions.
\end{abstract}

Keywords - livestock tracking architecture, Internet of Things, animal collar.

\section{INTRODUCTION}

$\mathrm{T}$ echnology in the mechanized agricultural sector around the world has been advancing at a steady pace to increase productivity and efficiency of different farmland processes. With the advances in Wireless Sensor Networks (WSN) and Internet of Things (IoT), there are endless potentials for their implementation in different domains, including habitat monitoring [1], wild animal tracking [2] and smart farming [3]. But the technology advances with respect to stock theft detection did not follow the same path. The simple reason would be that most developed countries don't need applications for this purpose, since cattle rustling occurs very rarely and in isolated occasions. However, in South Africa and Kenya it is certainly a huge problem for farmers with around 25000 cases reported in 2015 [4], [5].

Paper received April 24, 2018; revised October 8, 2018; accepted October 29, 2018. Date of publication December 25, 2018. The associate editor coordinating the review of this manuscript and approving it for publication was Prof. Aleksandar Nešković.

This work was carried out in the framework of COST Action CA15104 IRACON. The subject is supported by National Research Foundation, South Africa (grant numbers: IFR160118156967 and RDYR160404161474)

Biljana Risteska Stojkoska is with the Faculty of Computer Science and Engineering, Ss. Cyril and Methodius University, Skopje, R. Macedonia; (e-mail: biljana.stojkoska@finki.ukim.edu).

Dijana Capeska Bogatinoska is with the University of Information Science and Technology "St. Paul the Apostle" Partizanska bb, Ohrid, R.Macedonia; (e-mail: dijana.c.bogatinoska@uist.edu.mk).

Gerhard Scheepers is with the Department of Electrical, Electronic and Computer Engineering, University of Pretoria, South Africa.

Reza Malekian is with the Department of Electrical, Electronic and Computer Engineering, University of Pretoria, 0002, Pretoria, South Africa; (e-mail: reza.malekian@ieee.org).
From 2006 until 2010 around 377000 animals were stolen in South Africa, which adds to around R1 billion lost in 4 years [6].

One way to reduce stock theft is to keep account of all the cattle on a farm by constantly mustering them and counting the cattle [7]. This is a very labor-intensive task, but farmers have found that if they do not keep track of all their cattle on a frequent basis, the thieves steal more and more cattle from their herds.

In this paper, we propose a new architecture for real-time wireless livestock tracking solution based on Internet of Things. Additionally, we developed a new low-cost device for automatic livestock tracking in an outdoor environment (AnTrack) based on radio frequency (RF) technologies. AnTrack is designed as collar to be worn by the animals form the herds. It is self-sustainable with a watertight solar panel(s) capable of generating enough power to supply the device even when there is no sunshine for a week.

This paper is organized as follows. In the second section, a brief related work is provided regarding available solutions for animal and livestock tracking. Section III presents the architecture of our IoT based solution for livestock tracking. Details about the animal tracking device (AnTrack) are given in Section IV. Wireless technologies used for communication are elaborated in Section $\mathrm{V}$. Section VI and VII present discussions, future directions and conclusion.

\section{RELATED WORK}

With the rapid development of wireless and embedded technologies, many new opportunities appear for monitoring and tracking mobile objects, including animals. The first WSN based systems for animal tracking were costly and were not operating in real-time. Just ten years ago, the cost of collar was around $800 \$$ and data were sent twice per day [8]. In the last years, the price of the hardware has dropped drastically, together with the advances in communication technology. The first GPS-based animal tracking systems used to store GPS locations locally, but modern approaches incorporate data transfer techniques to send data at regular intervals in real-time or near-real-time, using radio communication, global system for mobile communications (GSM), low-earth-orbit (LEO) satellites (Iridium and Globalstar) and ad-hoc networks [9], or even Bluetooth Low Energy (BLE) in the most recent studies [10]. New IoT based farming and animal tracking solutions collect data in real-time, therefore are facing big data challenges, like privacy or security issues, data quality, intelligent processing and analytics, as well as sustainable data integration [11]. 
wildCENSE is a WSN that monitors the behavior and migration patterns of Swamp Deer [12]. The node is developed using ATMega microcontroller, GPS receiver, rechargeable battery, as well as solar recharging mechanism. Real-time data transmission is achieved using multi-hop network with ZigBee communication protocol. A new light weight, solar powered livestock tracking telemetry ear tag was developed in [13]. It weighs less than $40 \mathrm{~g}$ and can be easily attached on avian, but also can be used for larger animals. For wireless connection for data retrieval, the tag uses available $868 \mathrm{MHz}$ SRD/ISM frequency band. Testing results show farthest transmission range of $1 \mathrm{~km}-2.4 \mathrm{~km}$, depending if the line of sight is impeded or not. A standalone base station communicates with all individual tags and collects GPS location in a local database, before periodically uploading the data to the central cloud using HTTP protocol. In [14], the authors developed a new, highly efficient and reliable wildlife tracking device. To extend the duration of wild life tracking, the collar is equipped with a multi-source energy harvester, i.e. a solar energy harvester and an innovative rotational electromagnetic energy harvester. In [15], the authors present the design of Camazotz platform, dedicated to monitor flying foxes. It is a complete energy harvesting animal tracking solution with solar-based mobile sensing nodes integrated into an animal collar. The GPS data are not sent in real-time, but stored locally. If there is a base station closer than $200 \mathrm{~m}$, the collar is able to send all recordings through radio-based communication. The authors identify the major challenges in designing animal tracking solutions as: (i) building a device that does not affect the behavior of the animal in its natural habitat, i.e. the tracking device should not weigh more than $5 \%$ of the animals' body weight; and (ii) meeting energy constraints, that are correlated with the size of the devices and with the high power consumption of the GPS receiver.

Regarding cow and cattle tracking, the authors in [16] use data collected from collar, halter and ear tag to classify cattle behavior. In [17], small, inexpensive GPS loggers are used to monitor cattle movements and to analyze the impact of cattle grazing on vegetation in central Kenya. In both studies [16] and [17], the analyses are performed offline. One recent study describes an IoT framework in Greece using LoRaWAN long range communications [18]. LoRaWAN is suitable for this purpose since it is a less power consuming solution and can cover areas up to $15 \mathrm{~km}$ $20 \mathrm{~km}$.

Only few studies in the literature consider the problem of theft prevention. A South African product called CelMax has been developed specifically for stock theft prevention of sheep. It works by placing a collar device around the neck of a single sheep in a herd of 500. Sheep usually follow the actions of the others; when one sheep behaves abnormally, all of them will. When the collar devices detect running at night then it is assumed that someone is chasing them, which could be a thief trying to catch sheep. The device then sends an SMS to the farmer to let him know that the sheep are running and probably being chased. The battery life of this device ranges from 6 to 8 weeks and the whole device is very expensive [19]. Another product called the ProTagTor system was launched in 2009 which works on the same principle as the CelMax system but uses base stations instead of onboard GSM module to send an SMS to a farmer [20]. Their signals are forwarded to the base stations for analysis. Each of these tags has a battery life of 4 to 7 years and costs $10 \%$ of the GSM collar.

\section{SYSTEM ARCHITECTURE FOR LIVESTOCK TRACKING}

In this section, we are describing a generic system architecture for real-time livestock tracking that can be applied for different wireless devices attached to the animals. It is given in Fig. 1 and consists of three main building blocks: sensor side (animal collars organized as Wireless Sensor Network), server side (cloud) and client

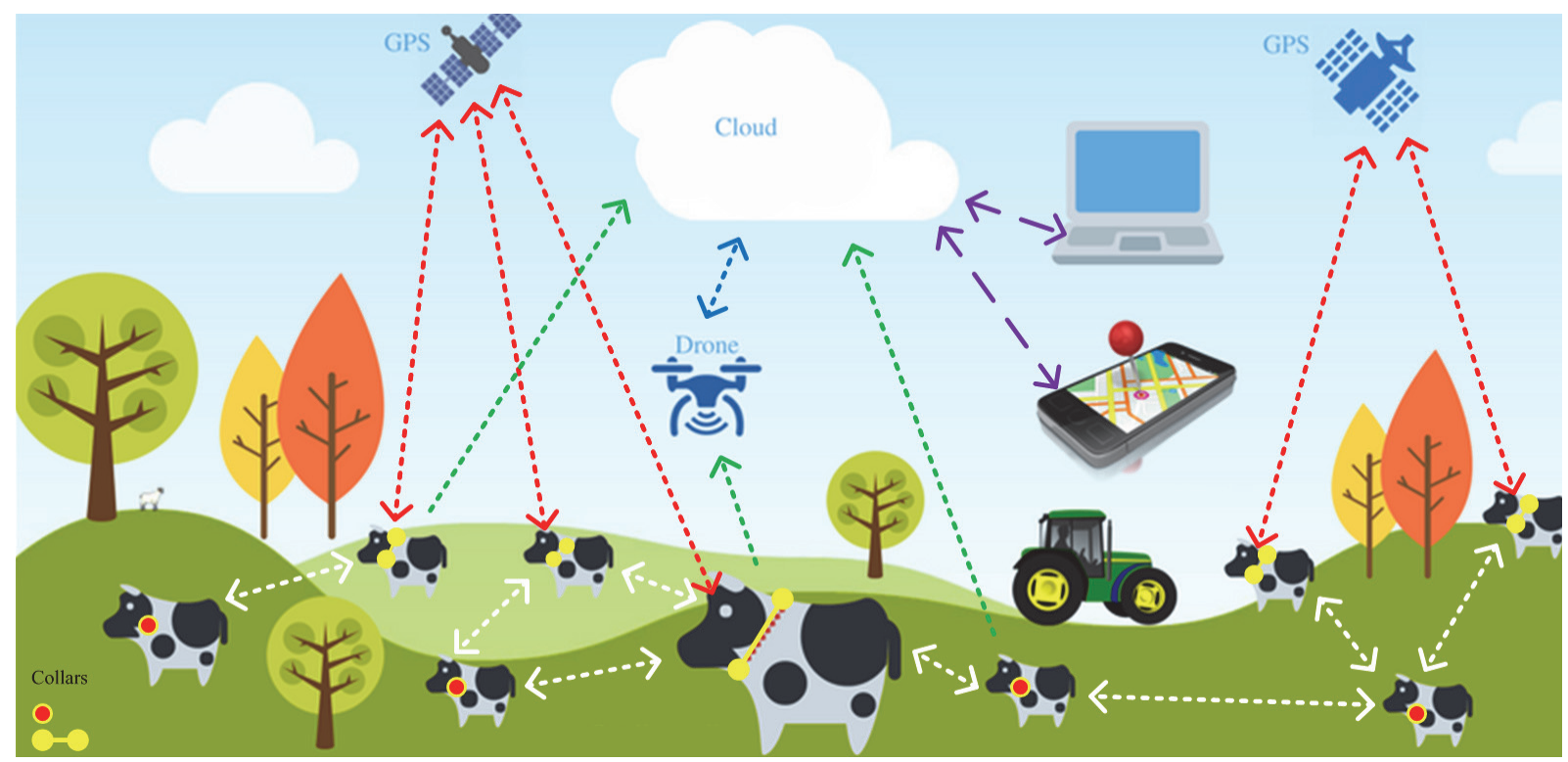

Fig. 1. System architecture for livestock tracking. 
side (represented by the end-users' computers or smartphones).

Animals in the herd are equipped with wireless collars. Those devices possess GPS interfaces capable to obtain the location of the animal. Since GPS sensors are expensive by means of energy consumption, they are not active all the time, but they switch between active and standalone mode, on predefined time intervals (those intervals are application specific and depend on the moving pattern of the animals being tracked). The GPS location needs to be sent to the base station (cloud) in real-time. For this purpose, the collar needs to be equipped with a module for wireless communication. The GPS data from the collar can be sent through different methods, including GSM or long-range communication protocols like LoRaWAN. In such a scenario, not all the animals need to be equipped with such modules. They can send GPS data directly to the animals in their close proximity (neighboring animal), so the data can be further resent to the nearest animal equipped with a module for wireless communication. For inter-animal communication, well known protocols like Zigbee, Z-Wave or BLE can be used. Not all collars need to have a module for obtaining GPS location. In order to save energy, some of the animals (collars) can apply algorithms to obtain their own location using the GPS data from the neighboring animal. Those techniques range from very simple like trilateration, to more sophisticated that apply complex optimization algorithms. Once they calculate their own location, they can share it with other collars or send it to the cloud.

Cloud is the central part of the architecture (Fig. 1). It gathers all data and performs more complex tasks regarding data analyses. With the rapid expansion of drone technology, it is also possible to use drones for data collection, where collars can send data directly to the drone using low cost protocols, while drone is responsible to resend the data to the cloud. In such a case, the drone acts as a hub or gateway between the collars and the cloud (Fig. 2). Clients (farmers and other interested parties) get feedback from the cloud through standard interfaces, like mobile and web applications.

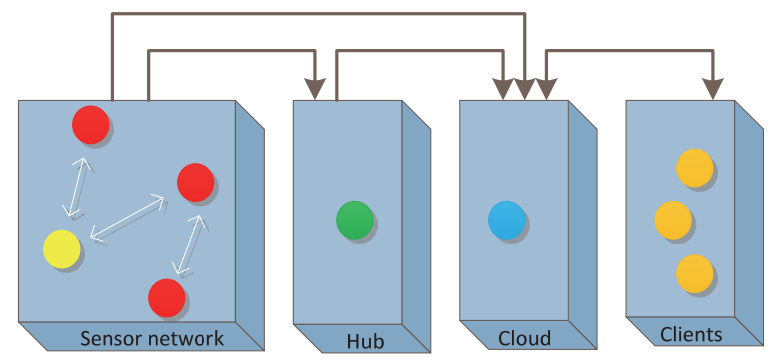

Fig. 2. Building blocks of the architecture with dataflows.

\section{ANTRACK DESIGN}

The three key modules of AnTrack are: a locator based on GPS, a solar panel to recharge the battery and a transmitter. The complete system will be in the form of a collar as seen in Fig. 3. The collar's strap will house a part of the flexible solar cells which will be wired to the device found underneath the animals' neck on the strap. The processor, as shown in Fig. 4, is an enclosure to house the electronics and battery in a watertight environment.

Fig. 4 shows the basic system diagram of the proposed device. The microcontroller unit (MCU) is responsible to control all other units connected to the system. The system contains two energy supply units: a battery and a solar panel. When the solar panel provides more power than the system needs, the power management subsystem charges the battery until it is fully charged. The system will rely exclusively on the battery if the solar panel is not providing enough power. The MCU controls the power supplied to the GPS module and RF transceiver. To keep power to a minimum the MCU uses its internal oscillators and should be in a sleep mode when the system is not recording the position or transmitting. The GPS module has a constant supply to its V Backup pin to ensure that SRAM is always being powered; the voltage will be stepped down to the specified range. The MCU has an external storage connected where location data will be saved when the base station is not in range, with the nRF option or if there is not enough GSM signal to transmit a location point using this method.
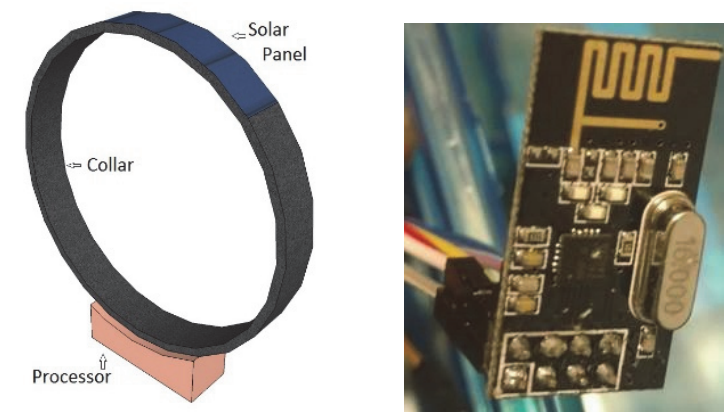

Fig. 3. Representation of AnTrack collar device.

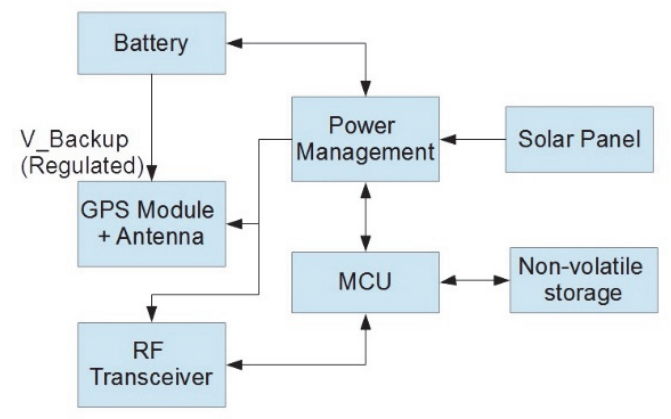

Fig. 4. AnTrack system diagram.

To complete this system, it is needed to use two different wireless technologies. One of them would be to find the location of the animal and the other wireless technology to use would be to transmit the data back to a server for analyses.

\section{A. GPS based tracking module}

GPS or Global Positioning Satellite has lots of different uses ranging from home to services. In AnTrack, the GPS module is used to track the animal in its habitat. The position is recorded by the microcontroller at an adjustable interval. 
The GPTA010 GPS module's datasheet [22] is used for additional information requirements. It runs with a voltage source from $3.0 \mathrm{~V}$ to $4.3 \mathrm{~V}$, typically $3.3 \mathrm{~V}$. At an update rate of $1 \mathrm{~Hz}$, to get a lock onto the position, the typical current consumption of this device is around $25 \mathrm{~mA}$, or power consumption at $82 \mathrm{~mW}$. While tracking, it uses $20 \mathrm{~mA}$ of current. For the purpose of this application, tracking is not necessary since the location is needed at 15 minutes.

TABLE 1: NMEA SHORT DESCRIPTION [21].

\begin{tabular}{|l|l|}
\hline NMEA Sentence & Description \\
\hline GGA & $\begin{array}{l}\text { Time, position and fix } \\
\text { type data }\end{array}$ \\
\hline GSA & $\begin{array}{l}\text { GPS receiver operating } \\
\text { mode, active satellites } \\
\text { used in the position } \\
\text { solution and DOP values }\end{array}$ \\
\hline GSV & $\begin{array}{l}\text { The number of GPS } \\
\text { satellites in view satellite }\end{array}$ \\
\hline $\begin{array}{l}\text { ID numbers, elevation, } \\
\text { azimuth, and SNR values }\end{array}$ & $\begin{array}{l}\text { Time, date, position, } \\
\text { course and speed data. }\end{array}$ \\
\hline RMC
\end{tabular}

TABLE 2: GGA SENTENCE STRUCTURE [21].

\begin{tabular}{|c|c|c|}
\hline Type & Example & Description \\
\hline Message ID & \$GPGGA & GGA protocol header \\
\hline UTC Time & 064951.000 & hhmmss.sss \\
\hline Latitude & 2307.1256 & ddmm.mmmm \\
\hline N/S Indicator & $\mathrm{N}$ & $\mathrm{N}=$ north or $\mathrm{S}=$ south \\
\hline Longitude & 12016.4438 & dddmm.mmmm \\
\hline E/W Indicator & $\mathrm{E}$ & $E=$ east or $W=$ west \\
\hline \multicolumn{3}{|l|}{ Position Fix } \\
\hline Indicator & 1 & 0 \\
\hline 1 & - GPS Fix & \\
\hline 2 & $\begin{array}{l}- \\
\text { Differential } \\
\text { GPS Fix }\end{array}$ & \\
\hline Satellites Used & 8 & Range 0 to 14 \\
\hline HDOP & 0.95 & $\begin{array}{l}\text { Horizontal Dilution } \\
\text { of Precision }\end{array}$ \\
\hline MSL Altitude & 39.9 & $\begin{array}{l}\text { Antenna Altitude } \\
\text { above/below mean- } \\
\text { sea-level }\end{array}$ \\
\hline Units & M & $\begin{array}{l}\text { Units of antenna } \\
\text { altitude }\end{array}$ \\
\hline $\begin{array}{l}\text { Geoidal } \\
\text { Separation }\end{array}$ & 17.8 & \\
\hline Units & $\mathrm{M}$ & $\begin{array}{l}\text { Units of geoids } \\
\text { separation }\end{array}$ \\
\hline
\end{tabular}

This module uses a Universal Asynchronous Receiver/Transmitter (UART) to communicate. The following is an example of how the GPS transmits data to a device connected via UART using the well-known NMEA protocol used by most GPS devices.

$\$ G P G G A, 064951.000,2307.1256, N, 12016.4438, E, 1,8,0$ $.95,39.9, M, 17.8, M,{ }^{*} * 5$

$\$ G P G S A, A, 3,29,21,26,15,18,09,06,10,,,,, 2.32,0.95,2.11$ *00

\$GPRMC,064951.000,A,2307.1256,N,12016.4438,E,0.0

\section{3,165.48,260406,3.05, $W, A^{*} 2 C$}

The NMEA sentences (see Table 1), are sent at a rate of $1 \mathrm{~Hz}$ but can be adjustable. Each sentence displays different information about the location, speed, time and satellite statuses. Table 2 sums up these sentences. \$GP is used as the header and not included in the table. We only require the GGA sentence because we only require the location.

\section{B. Data transmission module}

AnTrack can rely on two different methods to transmit position data to the server, based on GSM and RF technology respectively.

GSM based transmission: The GSM module is able to transmit an animal's paths constantly to a server using already available GSM network provided by cellular service providers. This method has its drawbacks. Each device needs a SIM card to operate and this SIM card needs to be loaded with data each time it nears its end of the data bundle. API's can be used for this process [23] which monitors each SIM and the server will be able to determine which SIM card needs recharging and adequately recharge the device with the correct data bundles. Quite important for these GSM modules related to power use is the fact that they can draw peak currents [21] of up to $2 \mathrm{~A}$, which requires that a sufficiently charged Lithium-ion battery be present. To communicate, this module uses the UART protocol. It uses the AT command protocol to communicate with an external device and the complete setup can be done using these commands. The GPRS module will need to establish connectivity to its cellular network.

When the GPRS module is in use and transmitting, the current being drawn can range from $156 \mathrm{~mA}$ to $408 \mathrm{~mA}$ depending on the number of RX/TX channels in use. To accurately predict the current consumption beforehand will be quite impossible which is why the worst-case scenario should always be assumed, $1.63 \mathrm{~W}$ at $4 \mathrm{~V}$ of power being used. The amount of time the module may search for its network and connect to the server can also be within quite a large range of seconds depending on the environment. A true range could not be found, and estimation will be made that the whole process may take around 1 minute to send a location to an HTTP server.

RF based transmission: The nRF $24 \mathrm{~L} 01 \mathrm{~L}$ is an RF transceiver operating at the $2.4 \mathrm{GHz}$ frequency, with a range of around $1.1 \mathrm{~km}$ and data rate at a maximum of $2 \mathrm{Mbps}$. This device is very feasible for this scenario. The only downside is that it requires a base station to receive the locations from the device. Its ultra-low current consumption of around $0.12 \mathrm{~mA}$ when transmitting [24], allows being almost exclusively powered by the solar panels except when operating at night. The device uses the SPI protocol to communicate with an MCU.

\section{Solar panel module}

Flexible solar panels are quite new in the industry but will be very useful in this application. The PowerFilm PT15-75 is $90 \mathrm{~mm}$ wide and $270 \mathrm{~mm}$ long, about the dimensions of the proposed collar strap [25]. It is water protected and sealed for outdoor use. In order to calculate if this solar panel is adequate for this application scenario, the 
expression (1) to calculate energy (E) has been adopted from [26] and [27]:

$$
E=A \cdot r \cdot H \cdot P R
$$

where $E$ is Energy ( $\mathrm{kWh} /$ year), $A$ is a total solar panel area $\left(\mathrm{m}^{2}\right)$ which is $0.0243 \mathrm{~m}^{2}, r$ is solar panel yield (\%) which is $3.16 \%, H$ is an annual average solar radiation on tilted panels (shadings not included) which is $2007 \mathrm{kWh} / \mathrm{m}^{2} /$ year, $P R$ is a performance ratio, coefficient for losses (range between 0.5 and 0.9 , default value $=0.75$ ).

As can be seen in Table 3, the power needed per day is only calculated at $23.99 \mathrm{mWh}$ (nRF24L01) [28], [29]. The solar panel which has been used to fit onto the collar will generate $2740 \mathrm{mWh}$ daily on average. For reliable operation of the AnTrack, the amount of power needed will be estimated to power the device for 5 days. This is a rough estimate where low light is possible due to heavy rainfall days and will be around $120 \mathrm{mWh}$ /day of power, backtracking this to the flexible solar panels [30] (using the panel sizes and current specifications, related that a solar panel with an estimate area size of $0.001 \mathrm{~m}^{2}$ is necessary or a solar panel of $1 \mathrm{~cm}$ by $1 \mathrm{~cm}$ ).

TABLE 3: POWER USAGE FOR DIFFERENT DEVICES

\begin{tabular}{|l|l|l|l|}
\hline Device (State) & $\begin{array}{l}\text { Power } \\
\text { Usage } \\
\text { [mW] }\end{array}$ & $\begin{array}{l}\text { Runtime } \\
\text { per } \\
\text { position } \\
\text { [s] }\end{array}$ & $\begin{array}{l}\text { Watt/hr } \\
\text { per day } \\
\text { [mWh] }\end{array}$ \\
\hline $\begin{array}{l}\text { GPS (Get } \\
\text { Position) }\end{array}$ & $82.5[7]$ & 10 & 22.917 \\
\hline $\begin{array}{l}\text { nRF24L01 } \\
\text { (Power Down } \\
\text { Mode) }\end{array}$ & $\begin{array}{l}0.0027 \\
{[9]}\end{array}$ & 870 & 0.065 \\
\hline nRF24L01(TX) & $33.9[9]$ & $1(1 \mathrm{Mb})$ & 0.942 \\
\hline $\begin{array}{l}\text { SIM800 (Power } \\
\text { Down) }\end{array}$ & $0.52[6]$ & 840 & 0.0225 \\
\hline SIM800 (Active) & $1630[6]$ & 60 & 2720 \\
\hline $\begin{array}{l}\text { PIC16F1508 } \\
\text { (RTC) }\end{array}$ & $\begin{array}{l}0.0009 \\
{[15]}\end{array}$ & 900 & 12.13 \\
\hline $\begin{array}{l}\text { PIC16F1508 (in } \\
\text { Use) }\end{array}$ & $\begin{array}{l}0.105 \\
{[15]}\end{array}$ & 15 & 0.044 \\
\hline $\begin{array}{l}\text { Total } \\
\text { (nRF24L01) }\end{array}$ & \multicolumn{2}{|c|}{} \\
\hline Total (SIM800) & & $\mathbf{2 4} \mathbf{m W h}$ \\
\hline
\end{tabular}

When looking at the SIM800 as an option, the total power required per day is $2.75 \mathrm{Wh}$. The solar panel can supply an average of $2.74 \mathrm{Wh}$ each day. This option is too close for comfort and the reliability of the system will not be good enough. To solve this, one solution is to use a large battery, but it will drain over time. Other option is to use a larger solar panel which will increase the size of the device. The last option to consider is to extend the intervals when locations are being sent to the server. When looking at Table 3, the MCU and GPS [31], [32] are used negligibly and when increasing the locations sent to every hour, the device will have a power consumption of 4 times less, suitable enough for the solar panel used.

\section{Discussions AND FUtURE DiRECTIONS}

This paper contains different power calculations when using two different transmitting devices which are around the same cost. These calculations have confirmed that the device can be self-sustainable by keeping its battery charged using small flexible solar panels. The biggest difference between the two communication methods is the fact that the GSM module can transmit from anywhere without any extra infrastructure and the nRF24L101 needs a base station which will only send the data to the server when within the $1 \mathrm{~km}$ specified range. However, the nRF24L101 uses a lot less power and it will almost be possible to manufacture the device in an ear tag form. The device is self-sustainable with a relatively small solar panel to keep its battery charged. The downfall of the GSM module is its large power consumption, it is so large that the other devices' consumption is negligible when transmitting at 15 minutes intervals, which means that the interval must be extended, and then base station solution is comparable as the two approaches can be set to send all the backed-up location data only once every day. Because of the probability to decrease the size of the complete system when using the nRF, this will be the better solution.

The following tests have to be conducted with the collar, the solar cell, and the GPS in a real-world scenario. Only the specified components and modules will be used for each test and after enough data has been captured to specify a plausible working system, the different modules will be combined. This method is preferred to determine any shortcomings the system might have in real-world scenarios as it will be quite hard to determine where the problems have occurred in the device malfunctions.

- For a week in summer and in winter, the flexible strip will be placed on the top of the strap to receive a rough estimate of how much energy is produced per day by this device.

- With a GPS module with a mounted chip antenna, the signal must be tested. It is recommended that the GPS antenna is pointed to the sky, but it is preferred that the module is placed inside a watertight enclosure with the MCU and battery with only 1 external connection to the solar cell. This test will determine if it is required that the GPS antenna is mounted on the solar cell to point to the top.

- Real world GPS tests will determine the amount of current that GPS uses and how long the system should run to lock onto a signal. This should determine at which interval the positions can be determined.

- Real world RF transmitter test will determine the current used to transmit a single as well as multiple positions to the base station. Because the base station and RF transmitter will not always be in range, tests should be conducted to determine how much current the device will use to check if the base station is in range.

\section{CONCLUSION}

The main contribution of this paper is the new architecture for wireless real-time livestock tracking in an outdoor environment. Additionally, we propose AnTrack, a collar-shaped device based on RF technologies. AnTrack is a self-sustainable device with a watertight solar panel(s) and 
aims to be a more cost-effective solution compared to traditional tracking devices. Through advanced calculations, we prove that AnTrack is capable to generate enough power to supply the device even when there is no sunshine for a week.

\section{REFERENCES}

[1] B. Stojkoska, and D. Davcev, "Web interface for habitat monitoring using wireless sensor network," in Wireless and Mobile Communications, 2009. ICWMC'09. Fifth International Conference on, pp. 157-162. IEEE, 2009.

[2] I. Kosović, I. Nižetić, and K. Fertalj, "Discovering the animal movement patterns using hidden Markov model." International Journal of Computer and Information Technology, no. 3 (2014): 508514.

[3] P. P. Jayaraman, A. Yavari, D. Georgakopoulos, A. Morshed, and A. Zaslavsky, "Internet of things platform for smart farming: Experiences and lessons learnt." Sensors 16, no. 11 (2016): 1884.

[4] S. H. Kim, D. H. Kim and H. D. Park: "Animal Situation tracking service using RFID, GPS, and sensors," in: Computer and Network Technology (ICCNT), 2010 Second International Conference on, pp. 153-156. IEEE Press, New York (2001).

[5] 2015 crime stats for South Africa: everything you need to know. Available: http://businesstech.co.za/news/government/99648/2015crime-stats-for-south-africa-everything-you-need-to-know/

[6] Zwane A.A., E. van Marle-Kster, B.J. Greyling, and N. Mapholi, A review, "Forensic DNA technology to meet the stock theft challenges in South Africa", SASAS - South African Society for Animal Science. Available: http://www.sasas.co.za/review-forensic-dna-technologymeet-stock-theft-challenges-south-africa

[7] GrainSA, "Safety hints on how to prevent livestock theft". Available: http://www.grainsa.co.za/safety-hints-on-how-to-prevent-ivestocktheft

[8] P. E. Clark, D. E. Johnson, M. A. Kniep, P. Jermann, B. Huttash, A. Wood, M. Johnson, C. McGillivan, and K. Titus, "An advanced, lowcost, GPS-based animal tracking system." Rangeland Ecology \& Management 59, no. 3 (2006): 334-340.

[9] S. M. Tomkiewicz, M. R. Fuller, J. G. Kie, and K. K. Bates, "Global positioning system and associated technologies in animal behaviour and ecological research." Philosophical Transactions of the Royal Society B: Biological Sciences 365, no. 1550 (2010): 2163-2176.

[10] J. Trogh, D. Plets, L. Martens, and W. Joseph. "Bluetooth Low Energy based location tracking for livestock monitoring," in 8th European Conference on Precision Livestock Farming (EC-PLF 2017), pp. 469-475. 2017.

[11] S. Wolfert, L. Ge, C. Verdouw, and M. J. Bogaardt, "Big data in smart farming-a review." Agricultural Systems 153 (2017): 69-80.

[12] V. R. Jain, R. Bagree, A. Kumar, and P. Ranjan. "wildCENSE: GPS based animal tracking system," in Intelligent Sensors, Sensor Networks and Information Processing, 2008. ISSNIP 2008. International Conference on, pp. 617-622. IEEE, 2008.

[13] B. Panckhurst, P. Brown, K. Payne, and T. C. A. Molteno, "Solarpowered sensor for continuous monitoring of livestock position," in Sensors Applications Symposium (SAS), 2015 IEEE, pp. 1-6. IEEE, 2015

[14] Y. Wu, L. Zuo, W. Zhou, C. Liang, and M. McCabe. "Multi-source energy harvester for wildlife tracking," in Active and Passive Smart Structures and Integrated Systems 2014, vol. 9057, p. 905704. International Society for Optics and Photonics, 2014.
[15] P. Sommer, B. Kusy, R. Jurdak, N. Kottege, J. Liu, K. Zhao, A. McKeown, and D. Westcott. "From the lab into the wild: Design and deployment methods for multi-modal tracking platforms." Pervasive and Mobile Computing 30 (2016): 1-17.

[16] A. Rahman, D. V. Smith, B. Little, A. B. Ingham, P. L. Greenwood, and G. J. Bishop-Hurley, "Cattle behaviour classification from collar, halter, and ear tag sensors." Information Processing in Agriculture (2017).

[17] J. M. Schieltz, S. Okanga, B. F. Allan, and D. I. Rubenstein, "GPS tracking cattle as a monitoring tool for conservation and management." African Journal of Range \& Forage Science 34, no. 3 (2017): 173-177.

[18] N. Zinas, S. Kontogiannis, G. Kokkonis, S. Valsamidis, and I. Kazanidis, "Proposed open source architecture for Long Range monitoring. The case study of cattle tracking at Pogoniani." (2017).

[19] Farmer's Weekly - Cellphones beat stock thieves. Available: http://www.farmersweekly.co.za/article.aspx?id=5795\&h=Cellphon es-beat-stock-thieves

[20] Security.co.za, GPS tags to protect rhino from poaching. Available: https://www.security.co.za/news/17087

[21] SIMCom, SIM800F-Hardware Design. Available: https://www.sos.sk/productdata/20/34/13/203413/sim800f _hardwar $\mathrm{e} \backslash$ design $\backslash v 1.00$.pdf

[22] GlobalTop Technology Inc, FGPMMOPA6C GPS Standalone Module Data Sheet. Available: https://cdnshop.adafruit.com/datasheets/GlobalTop-FGPMMOPA6CDatasheet-V0A-Preliminary.pdf

[23] Flickswitch Pty Ltd, Flickswitch. Available: http://www.flickswitch.co.za

[24] Nordic Semiconductor, Single Chip 2.4GHz Transceiver Product Specification.

Available:https:/www.sparkfun.com/datasheets/Components/SMD/ nRF24L01Pluss_Preliminary_Product_Specification_v1_0.pdf

[25] PowerFilm Solar, OEM solar modules. Available: http://www.powerfilmsolar.com/products/oem-comparison-chart/ photovoltaic-software.com.

[26] PHOTOVOLTAIC SOLAR ELECTRICITY DESIGN TOOLS Available: http://photovoltaic-software.com/PV-solar-energycalculation.php.

[27] Ganguli, Souvik, Singh, and Jasvir, "Estimating the solar photovoltaic generation potential and possible plant capacity," in Patiala, Engineering, Instrumentation and Engineering, Electrical and Sangrur, Technology. Vol.2, 253-260 (2010).

[28] J. Wannenburg, and R. Malekian, "Physical activity recognition from smartphone accelerometer data for user context awareness sensing", IEEE Transactions on Systems, Man and Cybernetics: Systems, to be published.

[29] Z. Wang, N. Ye, R. Malekian, F. Xiao, and R. Wang, "TrackT: accurate tracking of RFID tags with mm-level accuracy using firstorder Taylor series approximation", AD Hoc Networks, Elsevier, Vol.53, pp.132-144, 2016.

[30] Beyond, nanoWatt XLP eXtreme Low Power PIC Microcontrollers, Available: ww1.microchip.com/downloads/en/DeviceDoc/30009941F.pdf

[31] Z. Wang, N. Ye, R. Malekian, R. Wang, and P. Li,"TMicroscope: behavior perception based on the slightest RFID tag motion", Elektronika ir Elektrotechnika (Impact factor: 0.561), Vol.22, No.2, pp.114-122, 2016.

[32] X. Jin, J. Shao, X. Zhang, W. An, and R. Malekian, "Modeling of nonlinear system based on deep learning framework", Nonlinear Dynamics, Springer, Vol.84, No. 3, pp.1327-1340, 2016 